\title{
A New Method For Removing High-Density Salt and Pepper Noise Based On Decision-based Based Algorithm
}

\author{
Nan Yang, Shi Yan, Xueting Jing, Tianqin Cui and Xiaodong du \\ School of Information Science and Engineering, Lanzhou University, Lanzhou, China \\ nyang15@Izu.edu.cn;m51@163.com;jingxt15@Izu.edu.cn;cuitq15@Izu.edu.cn; duxd15@lzu.edu.cn;
}

Keywords: Median filtering; Processing structure; Iteration method;

\begin{abstract}
The thesis proposes an improved median filtering algorithm for the restoration of images that are highly corrupted by salt and pepper noise. The proposed algorithm (PA) replaces the noisy pixel with the median of the already processed pixel values by changing the processing structure and using iteration method to solve three specific situations when all the pixels in the selected window are corrupted by noise. It turns out that PA can efficiently remove the salt and pepper noise when the noise level is higher than $60 \%$. Simulation results are given to illustrate that PA can be applied to gray scale image denoising and outperforms the existing methods.
\end{abstract}

\section{Introduction}

Salt and pepper noise is the black and white light dark noise composed of image sensor, transmission channel, and it is often caused by cutting image. When corrupted by Salt and pepper noise, the image appears as pepper noise and salt noise [1]. It greatly reduces the quality of the image and it is quite necessary to remove noise from images before some subsequent processing, such as edge detection, image segmentation and object recognition [2].

It is well known that linear filter is not good at dealing with the salt and pepper noise. So the researchers begin to use nonlinear signal processing techniques. The standard median filter (SMF) mainly depend on fast sorting algorithm, and it has a large amount of computation due to requiring a lot of sorting work. At high noise density, SMFs often exhibit blurring for large window sizes and insufficient noise suppression for small window sizes. In order to improve the filtering effect, adaptive median filter (AMF) is proposed, in this method possible noisy pixels are marked and replaced by using median value, other pixels are unchanged [3]. The size of the window grows with the increase of the noise density to find a more appropriate value. This method performs well at low noise density. However, to settle higher density of noise, the size of the window should be increased, it resulting in a decrease in edge resolution [4]. Then the decision-based algorithm (DBA) is proposed where the noisy pixel is processed by the median filter while the noise-free pixel is left unchanged [3]. If the median value is 0 or 255, neighboring pixel is used for replacement, thus it produces streaking effect.

This paper proposes an improved method on the basis of median filtering and it retains more details while removing the noise and still has a good performance under high noise density levels. Decision-based method is used to identify the pixel, in order to make full use the information of the image, processing structure should be different. We mark the pixels which are surrounded by full of noise pixels in select window, the values of others are replaced by median value of noisy-free pixels in the selected window when the first scanning. Then, we scan the image for the second time to process marked pixels. Proposed algorithm (PA) replaces the marked pixels with the median of the already processed pixel values in the selected window by considering the boundary and the four vertices to ensure the quality of the image. If original image contains more black (0) and white (255) pixels or the noise density is really high, the above method is inefficient, we call this situation as noise death corner, shows as Fig. 2. PA uses an iterative algorithm which can make the center pixel and the surrounding signal pixel connect to each other maximumly to solve the above problem. Simulation results shows that the algorithm mentioned in this paper is better than the existing mainstream algorithms, especially in the high noise density. 


\section{Preliminaries}

Let I represents the original image with the size of $M \times N$ ( $\mathrm{M}$ is the number of rows, $\mathrm{N}$ is the number of columns). The observed noisy image and restored image are denoted by x and y. $I(i, j)$ represents the gray value of the original image at the point $(i, j) \cdot(1 \leq i \leq M),(1 \leq j \leq N)$. In the salt and pepper noise model, each pixel in an image has the probability of $p / 2$ being contaminated by either a salt(white) dot or a pepper $\operatorname{dot}($ black). Where $(0<p<1)$ : the equation is given by as

$$
x(i, j)=\left\{\begin{array}{cl}
0 & \text { with probability of } \mathrm{p} / 2 \\
255 & \text { with probability of } \mathrm{p} / 2 \\
I(i, j) & \text { with probability of } 1-\mathrm{p}
\end{array}\right.
$$

The decision-based method as follows:

$$
x \text { is a }\left\{\begin{array}{c}
\text { noise }- \text { free pixel, if } 0<x(i, j)<255 \\
\text { pixel with noise } x(i, j)=0 \text { or } x(i, j)=255
\end{array}\right.
$$

In this method, selected window which is a $3 \times 3,5 \times 5$, and $7 \times 7$, window can be selected and replaced [5]. we select $3 \times 3$ at this paper.

\section{Proposed Algorithm}

In this subsection, PA using decision-based method to detecting the salt and pepper noise and locate the noise pixel, assuming $w(i, j)$ as the window with the size of the $3 \times 3$ which take pixel $(i, j)$ as center, and $n(i, j)$ as the number of noise-free pixels belong to $w(i, j)$. Then three following cases are possible:

A: Pixels in the select window are not all noisy. In this subsection, in order to avoid the interference of noise as much as possible, the value of the center pixel should be replaced by the median of the surrounding noise-free pixel.

B: Pixels in the select window are all noisy. This is the first focus of the PA, because $\mathbf{n}(\mathbf{i}, \mathbf{j})=\mathbf{0}$. It is known that adjacent pixels have strong relationship with each other, traditional processing structure just have four pixels available shown as Fig. 1(a), it is obvious not enough, in order to make full use of the information of the image, we mark the pixel when first scanning, the value of current unknown pixel is calculated based on the adjacent pixels in the $\mathbf{w}(\mathbf{i}, \mathbf{j})$ during the next scanning and all of them have been processed, at that time there are eight pixels available shown in Fig .1(b). According to the center point location in the image, we process the noise pixels separately. When the noise pixels are locate in four edge of the image shown in Fig. 1, in order to avoid to introduce the noise which outside the scanning range, we should use median values of gray pixels in Fig. 1 to replace noisy pixel in the 1st boundary, other boundaries are treated similarly. If the noise pixels locate inside the image, the median value of gray pixels in Fig. 1(b) should use to replace.

$\mathrm{C}$ : Noise death corner. This is another focus of PA, assuming that all of the colored pixels are the noisy pixels as Fig. 2(1) shows, blue pixels are noise mentioned in case B and the gray pixels are adjacent to the signal pixels so that it can be process by case A. After the first scanning, as Fig. 2(1b)
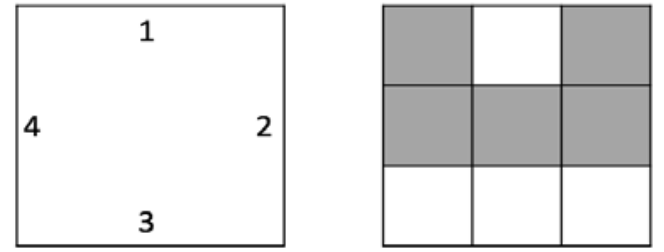

(a)

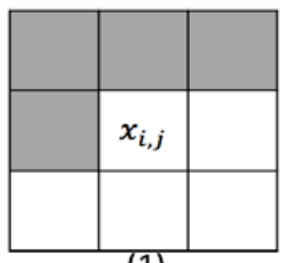

(1)

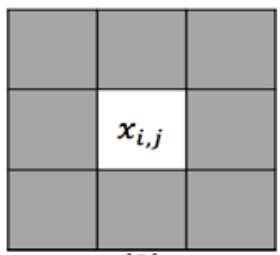

(2)

Figure 1. The death corner and Comparison graphs of PSNR and IEF at different noise densities for Lena image.

shows blue pixels are replaced by the median value of noisy pixel, so they are still noisy pixels, but the gray pixels have been processed, in order to solve the problem, the concept of iteration is introduced. Iterative method makes processing for many times. When the program run the second times, as Fig. 2(1c) shows the gray pixels of Fig. 2(1b) are replaced by the median value of white pixels and gray pixels in Fig. 2(1c) become treatable. By this way the blue pixels will all be processed. The probability of this situation is high so the iterative method is necessary to introduce. Depending 
on the noise density the number of iteration should be different. If the noise density is low, large numbers of iterations are not necessary. So the adaptive iterative method is added to ensure the program running more efficiency.

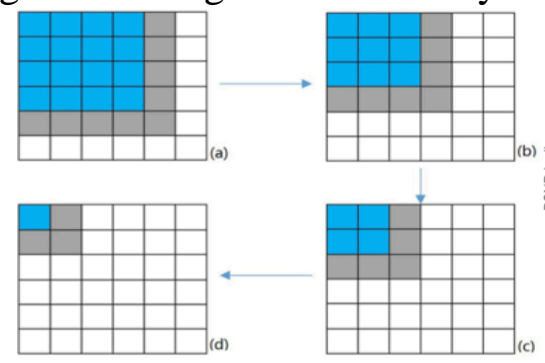

(1)

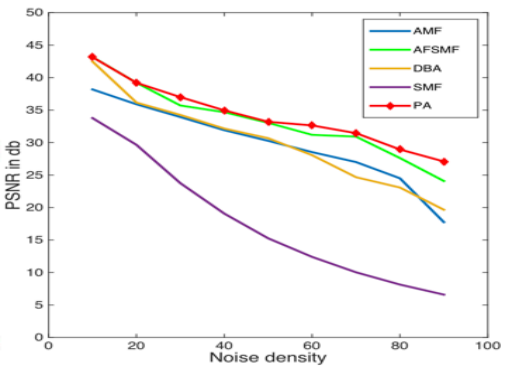

(2)

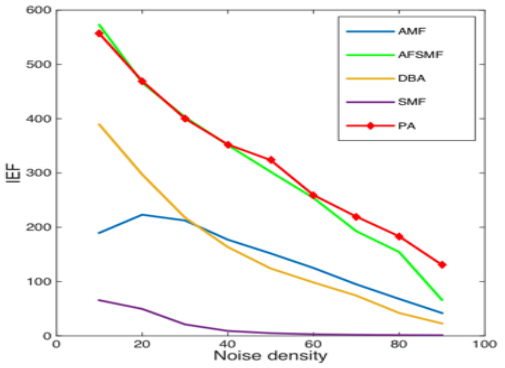

(3)

Figure 2. The death corner and Comparison graphs of PSNR and IEF at different noise densities for Lena image.

By following the above main idea, the algorithm is summarized as follows:

Step I: Scan the image and mark the pixels which are surrounded by full of noise pixels in select window. If $0<x(i, j)<255$, then $y(i, j)=x(i, j)$; else, output is median value of non-noisy pixels.

Step II: Scan the image for the second time to process marked pixels by using the value of processed pixels in select window.

Step III: Depends on different situations, use different iterative strategy.

Step IV: Output the final restored image.

\section{Simulations}

In this section, comparing based on Matlab experimental environment, and we choose Lena, Bridge, Man, Aerial as the experiment images to compare by AMF SMF AFSMF and DBA. We pollute the images with salt and pepper noise with different density from $10 \% \rightarrow 90 \%$. Considering two metrics peak signal to noise ratio (PSNR) and image enhancement factor (IEF) to assess the results of different algorithms objectively, which defined in (3) and (4) respectively, as

$$
\begin{aligned}
& P S N R=10 \log _{10} \frac{255^{2}}{M S E} \\
& I E F=\frac{\sum_{i}^{M} \sum_{j}^{N}\left(x_{i, j}-u_{i, j}\right)^{2}}{\sum_{i}^{M} \sum_{j}^{N}\left(y_{i, j}-u_{i, j}\right)^{2}}
\end{aligned}
$$

where MSE stands for mean square error.

$$
M S E=\frac{\sum_{i}^{M} \sum_{j}^{N}\left(u_{i, j}-y_{i, j}\right)^{2}}{M \times N}
$$

The result is shown in Table I and Fig. 2, PSNR and IEF values show that the proposed approach based on appropriate decision strategy can have a good output. All the algorithms have higher PSNR value at the low noise density. However, with the increase of the noise density, PA has the lowest reduce speed and perform stable in the high noise density. It also should be noted that, compared with AMF whose maximum window size is $39 \times 39$ in the above simulation, the PA uses a fixed $3 \times 3$ window for processing, which may lead to simple practical realization. IEF is similarity with PSNR. In order to prove PA which can performs well at different images, it is tested against gray scale images namely Man, Lena, Bridge and Aerial which are polluted by $70 \%$ salt and pepper noise. The PSNR values of these images using different algorithms are given in Table II. The visual performance of PA against the existing algorithms at noise densities of $70 \%$ and $90 \%$ for gray scale Lena image are shown in Fig. 2. It is observed that PA is better than other algorithms.

\begin{tabular}{|c|c|c|c|c|c|}
\hline \multirow{2}{*}{$\begin{array}{c}\text { Noise } \\
\%\end{array}$} & \multicolumn{5}{|c|}{ IEF of Lena } \\
\hline & $A M F$ & AFSMF & $D B A$ & SMF & $P A$ \\
\hline 10 & 189.75 & 572.59 & 389.17 & 65.64 & 556.85 \\
\hline 20 & 223.19 & 466.30 & 297.23 & 49.61 & 469.44 \\
\hline 30 & 212.49 & 402.90 & 217.85 & 21.11 & 400.29 \\
\hline 40 & 177.16 & 351.43 & 163.59 & 9.07 & 352.45 \\
\hline 50 & 151.86 & 302.26 & 124.13 & 4.95 & 302.79 \\
\hline 60 & 125.04 & 254.10 & 98.42 & 2.96 & 259.30 \\
\hline
\end{tabular}

Table 1. Perpormance (PSNR and IEF) of Different Algotithms For Lena Image At Different

Noise Density 


\begin{tabular}{|l|c|c|c|c|c|}
\hline 70 & 94.93 & 192.85 & 74.23 & 219.66 & 1.99 \\
\hline 80 & 68.13 & 154.53 & 42.15 & 1.49 \\
\hline 90 & 42.06 & 66.25 & 22.86 & 183.43 & 1.18 \\
\hline
\end{tabular}

\begin{tabular}{|c|c|c|c|c|c|}
\hline \multirow{2}{*}{$\begin{array}{c}\text { Noise } \\
\%\end{array}$} & \multicolumn{5}{|c|}{ PSNR(db) of Lena } \\
\hline & $A M F$ & AFSMF & $D B A$ & SMF & $P A$ \\
\hline 10 & 38.18 & 43.17 & 42.48 & 33.77 & 43.15 \\
\hline 20 & 35.90 & 39.21 & 36.16 & 29.65 & 39.20 \\
\hline 30 & 33.93 & 35.71 & 34.26 & 23.75 & 36.83 \\
\hline 40 & 31.91 & 34.68 & 32.16 & 19.07 & 34.96 \\
\hline 50 & 30.27 & 33.94 & 30.67 & 15.26 & 33.27 \\
\hline 60 & 28.53 & 31.19 & 28.06 & 12.40 & 32.69 \\
\hline 70 & 27.00 & 29.92 & 24.67 & 10.03 & 30.45 \\
\hline 80 & 24.50 & 28.62 & 23.08 & 8.14 & 28.94 \\
\hline 90 & 22.13 & 24.08 & 19.66 & 6.59 & 27.09 \\
\hline
\end{tabular}

Table 2. COMPARASION OF PSNR VALUES OF DIFFERENT TEST IMAGES AT NOISE DENSITY OF $70 \%$

\begin{tabular}{|c|c|c|c|c|c|}
\hline \multirow{2}{*}{ Test Images } & \multicolumn{5}{|c|}{ PSNR in $\mathbf{d b}$} \\
\hline & $A M F$ & AFSMF & $D B A$ & SMF & $P A$ \\
\hline Man & 25.78 & 28.25 & 25.06 & 9.45 & 28.79 \\
\hline Lena & 27.00 & 29.92 & 24.57 & 10.03 & 30.45 \\
\hline Bridge & 21.84 & 23.38 & 21.76 & 9.70 & 23.98 \\
\hline Aerial & 21.40 & 24.04 & 21.24 & 9.40 & 24.64 \\
\hline
\end{tabular}
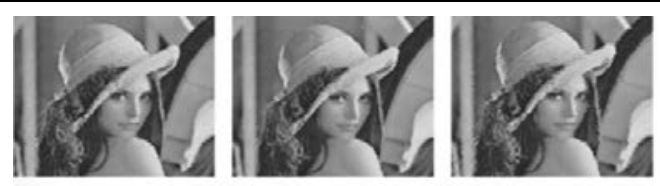

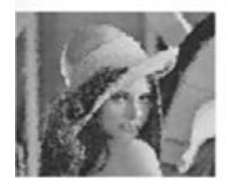

(a)

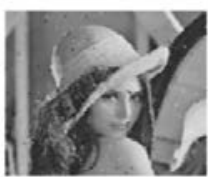

(b)

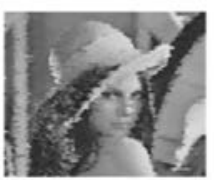

(c)

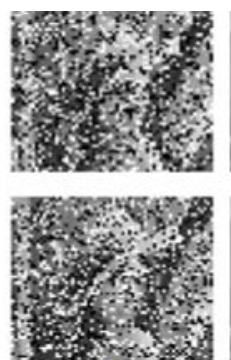

(d)

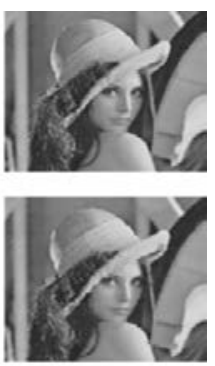

(e)

Figure 3. Performance of different algorithms for Lena corrupted by $70 \%$ salt and pepper noise in row $1,90 \%$ salt and pepper noise in row 2 . Outputs of (a) AMF (b) AFSMF (c) DBA (d) SMF (e) PA.

\section{Conclusions}

In this paper, an improved median filtering algorithm for the removal of high-density salt and pepper noise from images has been proposed. It has been shown that PA can remove noise effectively especially when the noise density is high. Simulation results have been given to show that the performance of PA is better than the existing algorithms for gray scale images with different textures and features.

\section{Acknowledgements}

This work was partly supported by the Fundamental Research Funds for the Central Universities (lzujbky-2016-136).

\section{References}

[1] A.Bovik, Handbook of image and video processing. New York: Aca- demic, 2000.

[2] Z.Wang and D. Zhang, "Progressive switching median filter for the removal of impulse noise from highly corrupted images,” IEEE Trans. Circuits Syst. II, vol. 46, no. 1, pp. 78-80, 1999.

[3] K.S.Srinivasan and D. Ebenezer, "A new fast and efficient decision- based algorithm for removal of high-density impulse noises,“ IEEE Signal Process. Lett. vol. 14, no. 3, pp. 189-192, 2007.

[4] I H.Hwang and R.A. Hadded, “Adaptive Median filter: New algorithms results," IEEE Transaction on image Processing, vol.4, no.4, pp. 499-S02.

[5] Chan, R.H., C.-W. Ho, and M. Nikolova, Salt-and-pepper noise removal. 